\title{
Preface to the Special Issue on Sexual Development and the Environment
}

\author{
Nicole Valenzuela ${ }^{\mathrm{a}}$ Manfred Schartl ${ }^{\mathrm{b}}$ \\ ${ }^{a}$ Department of Ecology, Evolution, and Organismal Biology, lowa State University, Ames, IA, USA; \\ ${ }^{b}$ Developmental Biochemistry, Biocenter, University of Würzburg, Würzburg, Germany
}

In sexually reproducing animals with separate sexes, sexual development involves the commitment of the developing embryo/larva to its sexual fate during the process of sex determination and the ensuing physical differentiation of the primary and secondary sexual organs/ traits. Sexual development results in the production of evolutionarily conserved phenotypes, males and females, yet this process shows a remarkable degree of evolutionary lability. Indeed, sexual development varies across the tree of life, even among vertebrates alone, and ranges in the degree of environmental plasticity underlying sex determination and differentiation. Thus, a continuum exists between sex-determining systems that are strongly canalized and under the control of sex chromosomes to systems under mostly environmental control. But even in species with sex chromosomes that are typically canalized, sex determination may be vulnerable to environmental influences under certain conditions, either commonly occurring in nature or rare anomalies in thermal regimes or ecotoxicological exposure.

This themed issue on "Sexual Development and the Environment" is dedicated to such phenomena, with the intention of bringing together a series of papers that review or present new theoretical or empirical data on ver- tebrate sexual development, either when the environment plays an integral part in the development of males and females, or when environmental conditions disrupt such processes.

In that vein, this volume opens with a review by Lisa E. Schwanz and Arthur Georges of 40 years of theory related to the ecology and evolution of environmental sex determination (ESD), thermolability, and the transitions between sex-determining mechanisms. Then, Nicole Valenzuela proposes a unifying explanation for the evolution of temperature-dependent sex determination (TSD) in long-lived and short-lived vertebrates, based on a review of ecological and genetic data from the Giant Amazonian turtle Podocnemis expansa. Next, Horacio Merchant-Larios, Verónica Díaz-Hernández, and Diego Cortez review the molecular and cellular mechanisms underlying TSD in turtles. This is followed by an empirical paper by Turk Rhen, Zachary Even, Alaina Brenner, Alexandra Lodewyk, Debojyoti Das, Sunil Singh, and Rebecca Simmons presenting an experimental study of Wnt signaling genes, their expression pattern in embryos of the snapping turtle Chelydra serpentina, and their molecular evolution across vertebrates. The ensuing review by Rachel M. Bowden and Ryan T. Paitz addresses the karger@karger.com www.karger.com/sxd

(c) 2021 S. Karger AG, Base

Karger!
Correspondence to:

Nicole Valenzuela, nvalenzu@iastate.edu

Manfred Schartl, phch1@biozentrum.uni-wuerzburg.de 
mechanisms by which maternal estrogens can affect the thermal responses of TSD turtle embryos to fluctuating temperature, with especial emphasis on Trachemys scrip$t a$, and the effect this can have on population sex ratios and evolution. Three papers then follow that focus on fish, a group where ESD and genotypic sex determination (GSD) coexist with species, and where sex reversal occurs commonly. First, the contribution by Carlos A. Strüssmann, Yoji Yamamoto, Ricardo S. Hattori, Juan I. Fernandino, and Gustavo M. Somoza reviews sex determination in atheriniform fishes, the coexistence and crosstalk between ESD and GSD in this group and how signals from both mechanisms are integrated. Second, Suzy C.P. Renn's and Peter L. Hurd's review focuses on cichlid fishes and how epigenetic regulation underlies a life-long battle between female- and male-determining molecular networks controlling gonadal and brain sexual development. Third, the review by Laura Casas and Fran Saborido-Rey describes recent advances illuminating the environmental cues that trigger typical sex change in hermaphroditic teleost fishes, and the molecular machinery deployed to detect and translate these signals during the transition between male and female phenotypes. The volume continues with a paper by Jonika Edgecombe, Lara Urban, Erica V. Todd, and Neil J. Gemmell, which explores the genomic basis of the plasticity underlying sex change/reversal present in some fish lineages and absent in others, and the potential role of gene duplication and neofunctionalization. Then, a group of papers covers other aspects of sex reversals. One of these papers, by Francesc Piferrer and Dafni Anastasiadi, reviews the occurrence of sex reversals across vertebrates and proposes that epigenetic inheritance may underpin the heightened environmental sensitivity exhibited by offspring of sex-reversed individuals. Another review by Sarah L. Whiteley,
Meghan A. Castelli, Duminda S.B. Dissanayake, Clare E. Holleley, and Arthur Georges discusses how the scarcity of examples of sex reversal in reptiles may be due to the paucity of studies in this area, and suggests approaches for their identification in the future. Then, the review by Adrián Ruiz-García, Álvaro S. Roco, and Mónica Bullejos argues that thermal sex reversals may be more common in amphibians than previously recognized, and may contribute to the turnover of sex-determining genes and the prevalence of homomorphic sex chromosomes in this group. Next, the paper by Yuta Sakae and Minoru Tanaka reviews examples linking metabolism and sex differentiation through experimental starvation in fish. The next group of papers address the effect of endocrine-disrupting chemicals (EDCs) in humans and other animals. First, Satomi M.N. Kohno reviews empirical evidence for the role of xenobiotics to induce sex reversals in crocodilians, thus altering sex ratios of wild populations. Then, Samuel M. Cripps, Deidre M. Mattiske, and Andrew J. Pask review endocrine signaling underpinning erectile function and explore the potential effects of EDCs in animals and humans. In closing, the review by Laura Gaspari, Benoit Tessier, Françoise Paris, Anne Bergougnoux, Samir Hamamah, Charles Sultan, and Nicolas Kalfa addresses the role of environmental EDCs on penile development in humans.

We hope this themed issue fosters further research into the effects that natural and altered environmental conditions have on sexual development and its evolution in vertebrates.

Nicole Valenzuela, Iowa State University, Ames, IA, USA

Manfred Schartl, University of Würzburg, Würzburg, Germany

June 2021 